\title{
Human Interaction with Passive Assistive Robots
}

\author{
Peng Pan, Kevin M. Lynch, Michael A. Peshkin and J. Edward Colgate \\ Laboratory for Intelligent Mechanical Systems \\ Mechanical Engineering Department \\ Northwestern University \\ Evanston, IL 60208
}

\begin{abstract}
We are studying the use of programmable constraint machines for rehabilitation and as assistive devices in materials handling. In this paper we describe initial experiments in human interaction with a two-joint passive programmable constraint device, or cobot. The user grasps the manipulandum at a handle, and the manipulandum implements a smooth, hard, low friction constraint curve. Initial experiments in reaching tasks subject to such a constraint show that subjects apply significant forces against the constraint, in a manner dependent on the movement speed and constraint shape. These forces can be broken into passive forces (due simply to the dynamics of the human arm) and forces actively generated by the muscles. Some motor adaptation is also evident.
\end{abstract}

\section{Introduction}

To design robots for human-robot collaborative manipulation, it is necessary to understand the human half of the system to ensure the safety of the operator and the overall effectiveness of the system. The design and control of human-interactive robots should take into consideration human motion preferences, ease and intuitiveness of the interaction, stress at joints, fatigue, etc. Our interest is in designing and controlling assistive robots to make manipulation of heavy loads faster, more comfortable, and less likely to result in work-related musculoskeletal disorders (WMSD's).

We are investigating the use of passive robotic guides to assist a human in manipulating a load from one configuration to another. A guide acts as a workless, frictionless rail which confines the load to an one-dimensional curve in its configuration space. A robot implementing a passive guide, for example a cobot [3], does not amplify human muscle power, but simply redirects the momentum of the load without affecting the energy. The presence of the guide assists by minimizing tedious fine positioning required of the human. In addition, the guide limits the object to one motion freedom; all other freedoms are force freedoms. This allows the operator to choose the most comfortable force combination in this space while the guide directs the

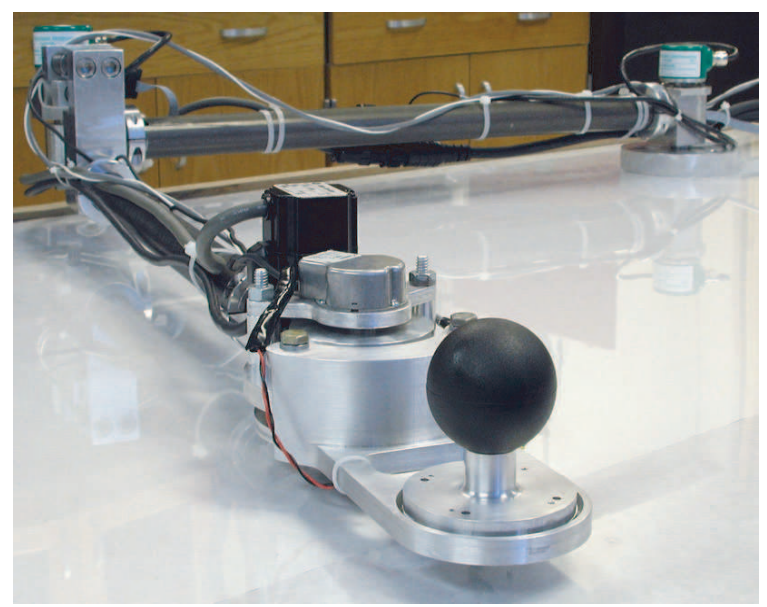

Fig. 1. A two-joint programmable constraint robot.

load to the goal. If certain muscles begin to fatigue, the operator may choose different force combinations, or the shape of the guide constraint can be modified. Since the robot guide is passive, it is as safe to interact with as a physical rail.

In this paper the programmable constraint machine is a planar 2R manipulandum capable of implementing smooth programmable guiding paths of arbitrary shape [4] (Figure 1). This cobot uses a computer-controlled rolling mechanism to make it possible to have simultaneously a high mechanical impedance in one direction and a low impedance in another direction - the feeling of a mechanical constraint, such as moving along a smooth rail, yet fully programmable.

This device was designed to investigate how humans naturally interact with kinematic constraints and to implement reaching motion rehabilitation protocols. In this paper we focus on the former topic and investigate speed and force profiles as subjects perform planar reaching motions along programmed curves. Understanding how subjects naturally apply forces against kinematic constraints during 
motion both provides insight into the organization of the motor control system and informs the design of constraint surfaces for repetitive materials handling tasks. In repetitive materials handling tasks, we believe the more kinematic constraints the better, provided the constraints are the "right" constraints for the user. Understanding what "right" means is one motivation for this work.

In previous work we studied static single-arm force generation with kinematic constraints [1]. The results showed that subjects apply significant constraint forces, and this behavior can be interpreted in terms of an objective function describing how subjects choose a particular hand force from an infinite set of hand forces that accomplish the task. This paper reports preliminary results of ongoing experiments where subjects perform reaching tasks subject to path constraints. The results show that subjects consistently apply significant forces against the constraints, dependent on the speed of motion and the shape of the constraint. There also appears to be motor adaptation; the interaction forces change depending on the history of paths followed. Efforts to quantify these effects are ongoing.

\section{Manipulation Forces}

The design of effective guides requires an understanding of how humans naturally manipulate a load confined to a constraint surface. Consider the grasped object as having six degrees of freedom, three translational and three rotational. We can choose six coordinates, for example $x$ $y$-z-roll-pitch-yaw, to represent the configuration of the object. (All choices of three coordinates for orientation will have singularities, but we will set aside this problem for ease of discussion.) Let the coordinate vector $\mathbf{q}$ be written as a column vector. The object is subject to a set of $k$ independent configuration constraints, expressed as the vector equation

$$
\mathbf{h}(\mathbf{q})=\mathbf{0}, \quad \mathbf{h}(\mathbf{q})=\left(h_{1}(\mathbf{q}), \ldots, h_{k}(\mathbf{q})\right)^{T} .
$$

Therefore, the object is constrained to move on a $(6-k)$ dimensional surface. Since these constraints are preserved, their time-derivatives are also zero,

$$
\frac{\partial \mathbf{h}(\mathbf{q})}{\partial \mathbf{q}} \dot{\mathbf{q}}=\mathbf{A}(\mathbf{q}) \dot{\mathbf{q}}=\mathbf{0},
$$

where $\mathbf{A}(\mathbf{q})$ is a $k \times 6$ matrix. The constraints are frictionless, so any generalized reaction force $\mathbf{f}_{r} \in \mathbb{R}^{6}$ (including forces and torques) acting on the load due to the constraints must be normal to the constraints, i.e., of the form

$$
\mathbf{f}_{r}=\mathbf{A}^{T}(\mathbf{q}) \boldsymbol{\lambda}, \quad \boldsymbol{\lambda} \in \mathbb{R}^{k} .
$$

The generalized force $\mathbf{f}_{r}$ lives in the $k$-dimensional space $\mathcal{F}_{n w}(\mathbf{q})$ of forces that do no work on the object. The rows of $\mathbf{A}(\mathbf{q})$ form a basis for this space. The $(6-k)$ dimensional space $\mathcal{F}_{w}(\mathbf{q})$ of forces that can do work on the object is orthogonal to $\mathcal{F}_{n w}(\mathbf{q})$ by the inertia metric (sometimes called the kinetic energy metric) defined by the object's $6 \times 6$ configuration-dependent inertia metric $\mathbf{M}(\mathbf{q})$, where the kinetic energy of the object is given by

$$
K=\frac{1}{2} \dot{\mathbf{q}}^{T} \mathbf{M}(\mathbf{q}) \dot{\mathbf{q}}
$$

In other words, for any $\mathbf{f}_{w} \in \mathcal{F}_{w}(\mathbf{q})$ and $\mathbf{f}_{n w} \in \mathcal{F}_{n w}(\mathbf{q})$, the following condition is satisfied:

$$
\mathbf{f}_{n w}^{T} \mathbf{M}^{-1}(\mathbf{q}) \mathbf{f}_{w}=0 .
$$

Note that this definition of orthogonality is different than the usual Euclidean definition of orthogonality, where the inertia matrix is replaced by the identity matrix.

Any generalized force $\mathbf{f}_{h}$ that the subject applies to the object can be written as the sum of working and workless forces, $\mathbf{f}_{h}=\mathbf{f}_{w}+\mathbf{f}_{n w}$. This decomposition is given by the projections

$$
\mathbf{f}_{w}=\mathbf{P}(\mathbf{q}) \mathbf{f}_{h}, \quad \mathbf{f}_{n w}=(\mathbf{I}-\mathbf{P}(\mathbf{q})) \mathbf{f}_{h},
$$

where $\mathbf{I}$ is the $6 \times 6$ identity matrix and $\mathbf{P}(\mathbf{q})$ is

$$
\mathbf{P}=\mathbf{I}-\mathbf{A}^{T}\left(\mathbf{A} \mathbf{M}^{-1} \mathbf{A}\right)^{-1} \mathbf{A} \mathbf{M}^{-1} \text {. }
$$

The subject has $k$ force freedoms (force directions that do not affect the motion) and $6-k$ motion freedoms, independent of any internal motion freedoms due to redundancy of the arm-body system.

\section{The Experimental Setup}

In this paper the "object" grasped by the human is a vertical-rod handle at the endpoint of a $2 \mathrm{R}$ robot arm in the horizontal plane. This handle can spin freely about a vertical axis. The $x-y$ direction of motion of the robot endpoint in the plane is determined by a computer-controlled steering wheel near the handle. According to the previous development, the object has four constraints at any time (three forcing it to be in the plane and one forcing it to be on a computer-controlled $x-y$ curve). The two motion freedoms are spinning of the handle and motion of the handle along the programmable curve. The subject grasps the handle with the right hand, with the wrist immobilized by a cuff and the shoulder location fixed. This allows us to treat the subject's arm as a 2-DOF mechanism. For this reason, we treat both the object and the human arm as 2DOF systems $(x-y$ position of the handle, joint angles for the human) subject to one motion constraint determined by the angle of the wheel.

Because the robot is a $2 \mathrm{R}$ mechanism, the apparent mass matrix of the handle changes with configuration. Let the handle configuration be written $\mathbf{q}=(x, y)^{T}$, with mass matrix $\mathbf{M}(\mathbf{q})$. For this system, a basis vector for the space of workless forces $\mathcal{F}_{n w}(\mathbf{q})$ at $\mathbf{q}$ is given by 


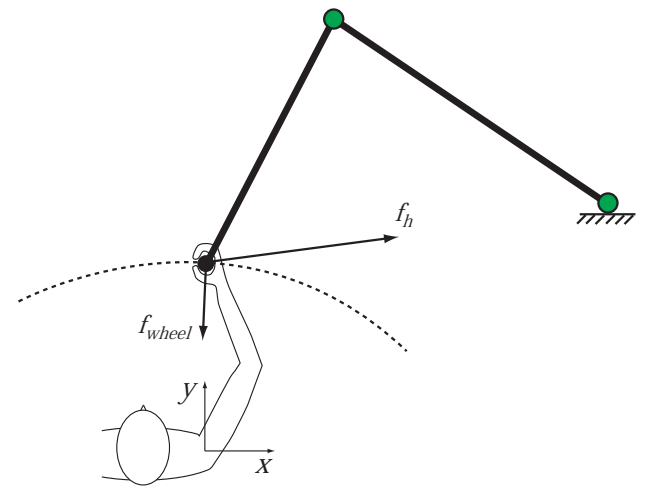

Fig. 2. Forces applied on the passive manipulandum.

$\mathbf{f}_{n w}=(\dot{y},-\dot{x})^{T}$. A basis vector for $\mathcal{F}_{w}(\mathbf{q})$ is any vector $\mathbf{f}_{w}$ that solves

$$
\mathbf{f}_{n w}^{T} \mathbf{M}^{-1}(\mathbf{q}) \mathbf{f}_{w}=0,
$$

such as $\mathbf{f}_{w}=\mathbf{M}(\mathbf{q}) \dot{\mathbf{q}}$. Let $\hat{\mathbf{f}}_{w}$ and $\hat{\mathbf{f}}_{n w}$ be the unit vectors of $\mathbf{f}_{w}$ and $\mathbf{f}_{n w}$ by the inertia metric, given by

$$
\hat{\mathbf{f}}_{*}=\frac{\mathbf{f}_{*}}{\sqrt{\mathbf{f}_{*}^{T} \mathbf{M}^{-1}(\mathbf{q}) \mathbf{f}_{*}}},
$$

where ' $*$ ' is either $w$ or $n w$. Then any force applied by the human can be written

$$
\mathbf{f}_{h}=\mu_{w} \hat{\mathbf{f}}_{w}+\mu_{n w} \hat{\mathbf{f}}_{n w},
$$

where $\mu_{w}$ and $\mu_{n w}$ are signed magnitudes.

Note that if the mass matrix did not change with the configuration, as with a point mass, then $\hat{\mathbf{f}}_{w}$ and $\hat{\mathbf{f}}_{n w}$ would satisfy Euclidean orthogonality, $\hat{\mathbf{f}}_{w}^{T} \hat{\mathbf{f}}_{n w}=0$, and the normalization to unit vectors would be the familiar Euclidean normalization. We could also use the word "tangential" to describe the forces that do work.

Two forces act on the "object" or manipulandum: a constraint force from the rolling wheel, $\mathbf{f}_{\mathrm{whee}}$, and the force applied by the human measured by the force sensor, $\mathbf{f}_{h}$ (Figure 2). We would like to know how much of $\mathbf{f}_{h}$ is due simply to the arm acting as a passive massdamper, with configuration-dependent mass, and how much is actively generated by the muscles. The total force applied by the human can be written

$\mathbf{f}_{h}=\mathbf{f}_{h, w, \text { passive }}+\mathbf{f}_{h, w, \text { active }}+\mathbf{f}_{h, n w, \text { passive }}+\mathbf{f}_{h, n w, \text { active }}$,

where $\mathbf{f}_{h, w}$,passive is the natural damping of the arm acting to remove energy from the manipulandum, $\mathbf{f}_{h, w}$,active are the forces applied by the muscles to add or subtract energy from the manipulandum, $\mathbf{f}_{h, n w \text {, passive }}$ are the passive forces of the arm due to its changing inertia that do no work on the manipulandum, and $\mathbf{f}_{h, n w}$, active are the forces actively applied by the muscles that do not change the energy of the manipulandum.
To distinguish these different components of $\mathbf{f}_{h}$, we must adopt a dynamic model of the subject's arm, including the inertial parameters, link lengths, and joint viscous friction coefficients. We adopted parameters from Uno et al. [2]. With these parameters, the equations of motion for the subject's arm can be written

$$
\boldsymbol{\tau}-\mathbf{b} \dot{\boldsymbol{\theta}}-\mathbf{J}^{T}(\boldsymbol{\theta}) \mathbf{f}_{h}=\mathbf{M}_{\mathrm{arm}}(\boldsymbol{\theta}) \ddot{\boldsymbol{\theta}}+\mathbf{C}(\boldsymbol{\theta}, \dot{\boldsymbol{\theta}}) \dot{\boldsymbol{\theta}},
$$

where $\tau$ is a 2 -vector of joint torques actively generated by the muscles, $\mathbf{b}$ is a 2 -vector of viscous friction coefficients at the joints, $\boldsymbol{\theta}$ is a 2 -vector of joint angles, $\mathbf{J}(\boldsymbol{\theta})$ is the subject's arm Jacobian satisfying $\dot{\mathbf{q}}=\mathbf{J}(\boldsymbol{\theta}) \dot{\boldsymbol{\theta}}, \mathbf{M}_{\text {arm }}(\boldsymbol{\theta})$ is a $2 \times 2$ symmetric, positive-definite inertia matrix, and $\mathbf{C}(\boldsymbol{\theta}, \dot{\boldsymbol{\theta}})$ is a $2 \times 2$ Coriolis matrix. Given our model of the subject's arm, the force sensor data, and the arm joint velocities and accelerations derived from the arm's kinematics and the manipulandum's encoders, we can solve (3) for the actively generated muscle torques $\tau$. These are transformed to forces at the hand by

$$
\mathbf{f}_{h, \text { active }}=\left(\mathbf{J}^{T}(\boldsymbol{\theta})\right)^{-1} \boldsymbol{\tau}
$$

which can be projected to working and nonworking forces. The remaining force, $\mathbf{f}_{h}-\mathbf{f}_{h}$,active, is classified as $\mathbf{f}_{h \text {,passive, }}$, and similarly split into a working and nonworking component.

Our primary interest in this paper is the relative magni-

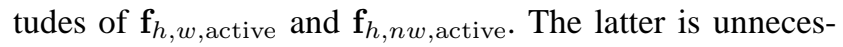
sary for the task; its sum with the constraint force provided by the wheel is constant regardless of how the subject chooses it.

\section{Preliminary Results}

We carried out preliminary studies with two subjects (healthy right-handed males). Subjects were seated in a custom-made high-backed chair with an adjustable seat, to raise or lower the height of the shoulder plane based on the height of the subject. To fix the shoulder location, subjects were restrained by a four-point harness. The wrist was immobilized by an over-the-counter commercially available wrist cuff, and the subject grasped a vertical handle mounted on the endpoint of the robot manipulandum (Figure 2). The handle can spin freely about a vertical axis so that no torques at the hand are involved, and a support plate is attached to the handle to support the weight of the forearm. This support maintains the arm in a horizontal plane throughout experiments without fatiguing the shoulder. The $x-y$ frame is aligned with the human shoulder frame. The robot manipulandum is mounted at $(0.7,0.9)$ and constrained to move in the same plane. In the experiments, we asked the subjects to move the endpoint of the manipulandum from a fixed start point $(-0.071,0.379)$ to a fixed end point $(0.071,0.521)$ along 


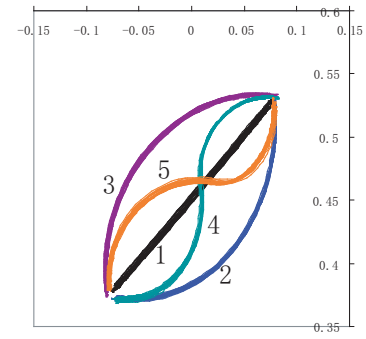

(a) Paths No. 1 through No. 5.

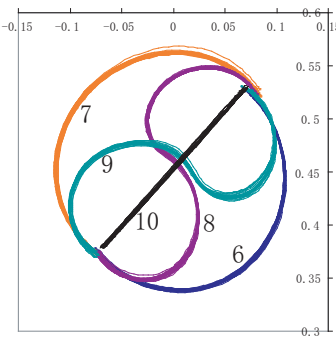

(b) Paths No. 6 through No. 10.

Fig. 3. All 10 constraint paths used in the experiments.

different constrained paths as fast as possible. A successful movement was defined as a movement that finished in a predefined time limit (different for each path). After subjects completed 20 successful movements along one path, the path was automatically switched to the next one. In total there were 10 paths (Figure 3). The first path (No. 1) and the last path (No. 10) are the same straight line. Other paths (No. 2 through No. 9) are curves with zero or one inflection point.

A six-axis force-torque sensor (ATI-AI Gamma 15-50) is positioned between the handle and the endpoint of the robot manipulandum. Two encoders mounted on the robot arm joints are used to record joint angles, which can be transformed to endpoint position. During each movement, both force and position data are recorded at a sampling frequency of $100 \mathrm{~Hz}$.

The protocol was approved by the Northwestern University Institutional Review Board.

\section{A. Active Workless Force}

Forces applied by subject 1 at the force sensor $\left(\mathbf{f}_{h}\right)$ during 20 movements along paths No. 2 and No. 9 are shown in Figures 4 and Figure 5, including (a) the active force component $\mathbf{f}_{h, \text { active }}$ and (b) the passive force component $\mathbf{f}_{h \text {,passive. }}$ Grey lines are working forces $\mathbf{f}_{h, w \text {,active }}$ and

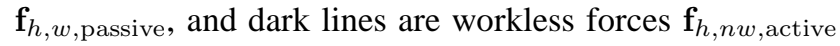
and $\mathbf{f}_{h, w \text {,passive }}$.

The figures show that the subject's interaction forces

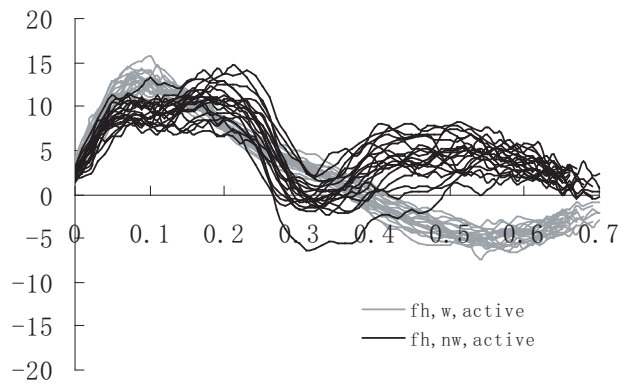

(a) Active forces.

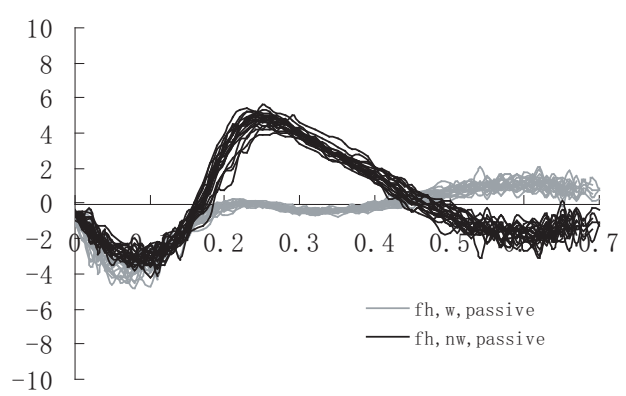

(b) Passive forces.

Fig. 4. Force-sensor-measured human applied forces during 20 movements along path No. 2.

are relatively consistent over the 20 motions. The active workless forces $\mathbf{f}_{h, n w \text {,active }}$ are significantly nonzero. This shows that the subject consistently applies force against the constraint even though it is unnecessary for the task. These constraint forces change according to subject's movement speed and constraint shape.

It is also worth noting that the average magnitude of active workless forces is as large as the average magnitude of active working forces. This is consistent with our previous results showing that subjects apply significant constraint forces in certain static force-balance tasks [1]. The active constraint forces we measured in our dynamic reaching tasks (after subtracting the constraint forces due to the significant passive dynamics of the arm) cannot be explained by simply applying the static configurationdependent-only results of our previous study, however, suggesting that the strategy for using constraints is velocity dependent.

\section{B. Motor Adaptation}

The first path (No. 1) and the last path (No. 10) used in the experiments are the same straight path. Subject 1's active forces $\mathbf{f}_{h, \text { active }}$ during 20 movements along paths No. 1 and No. 10 are shown in Figures 6 and 7. Grey lines

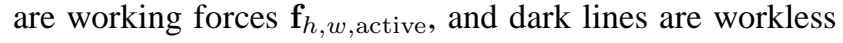




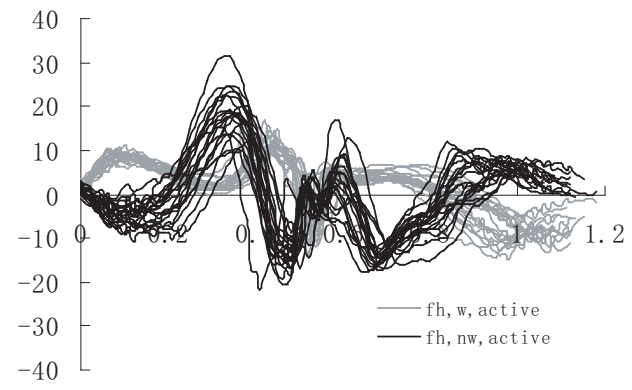

(a) Active forces.

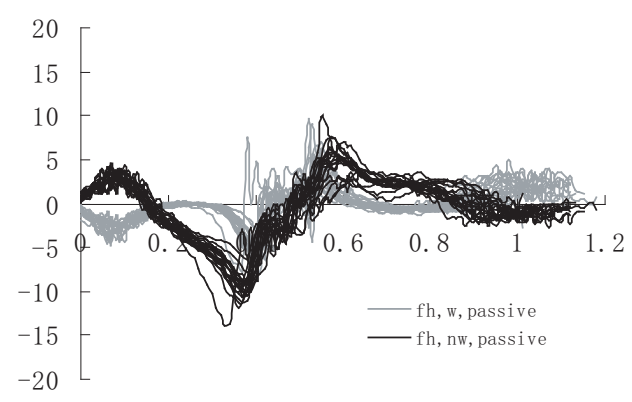

(b) Passive forces.

Fig. 5. Force-sensor-measured human applied forces during 20 movements along path No. 9.

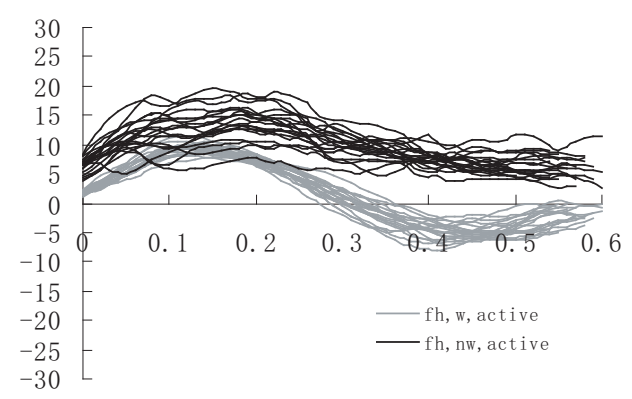

Fig. 6. Human applied active forces during 20 movements along path No. 1.

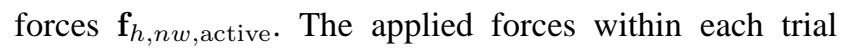
are similar, but there is a marked difference between the workless active forces. This suggests that reaching motions with the intervening 8 paths causes a change in the motor strategy. We are just beginning to explore the mechanism and explanation for this motor adaptation.

\section{Discussion and Ongoing Work}

In this paper, we presented a new type of passive assistive robot for characterizing normal subjects' interaction with passive constraints and for possible future use in quantify-

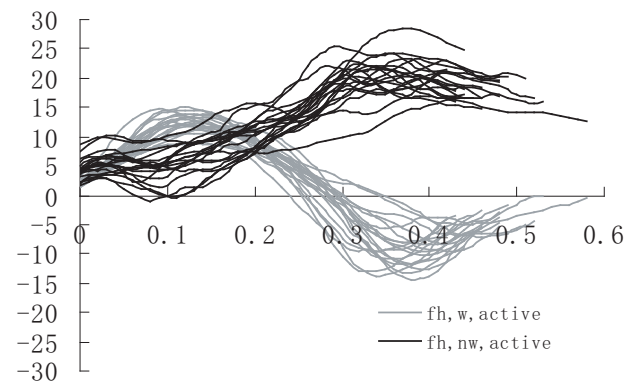

Fig. 7. Human applied active forces during 20 movements along path No. 10.

ing the deficit in stroke patients and implementing rehabilitation protocols. The virtual constrained paths (Figure 3) realized by the robot manipulandum are quite consistent from trial to trial. This demonstrates the robot's ability to reliably implement path constraints.

We modeled the dynamics of human interaction with the robot and decomposed the interaction forces between the human and robot as passive and active forces, and working and workless forces. We also presented the results of some preliminary experiments. The results so far show that subjects generate significant active workless forces in a way that cannot be explained by our static results, and that these active workless forces may be modified over time. Experiments with more subjects are ongoing. Our goal is to develop a predictive model of the workless forces actively applied by a subject as a function of the powering forces being applied by the subject, the configuration of the subject's arm, the speed and direction of motion, and the curvature of the path.

One goal of this line of work is to design constraint surfaces to assist a human in manipulating a load from one configuration to another. Constraint surfaces are passive and inherently safe to interact with, and a properly designed constraint surface or guide rail can improve the ergonomics of a repetitive material handling task.

\section{References}

[1] P. Pan, M. A. Peshkin, J. E. Colgate, and K. M. Lynch. Static single-arm force generation with kinematic constraints. Journal of Neurophysiology, doi:10.1152/jn.00799.2004.

[2] Y. Uno, M. Kawato, and R. Suzuki. Formation and control of optimal trajectory in human multijoint arm movement: Minimum torque change model. Biological Cybernetics, 61:89-101, 1989.

[3] W. Wannasuphoprasit, R. B. Gillespie, J. E. Colgate, and M. A. Peshkin. Cobot control. In IEEE International Conference on Robotics and Automation, pages 3571-3577, 1997.

[4] T. Worsnopp, M. A. Peshkin, J. E. Colgate, and K. M. Lynch. Controlling the apparent inertia of passive human interactive robots. In Proceedings of the 2004 IEEE International Conference on Robotics and Automation, volume 2, pages 1179-1184, 2004. 